\title{
3D Discrete Shearlet Transform and Video Processing
}

\author{
Pooran Singh Negi and Demetrio Labate
}

\begin{abstract}
In this paper, we introduce a digital implementation of the 3D shearlet transform and illustrate its application to problems of video denoising and enhancement. The shearlet representation is a multiscale pyramid of well-localized waveforms defined at various locations and orientations, which was introduced to overcome the limitations of traditional multiscale systems in dealing with multidimensional data. While the shearlet approach shares the general philosophy of curvelets and surfacelets, it is based on a very different mathematical framework which is derived from the theory of affine systems and uses shearing matrices rather than rotations. This allows a natural transition from the continuous to the digital setting and a more flexible mathematical structure. The 3D digital shearlet transform algorithm presented in this paper consists in a cascade of a multiscale decomposition and a directional filtering stage. The filters employed in this decomposition are implemented as finite-length filters and this ensures that the transform is local and numerically efficient. To illustrate its performance, the $3 \mathrm{D}$ Discrete Shearlet Transform is applied to problems of video denoising and enhancement, and compared against other state-ofthe-art multiscale techniques, including curvelets and surfacelets.
\end{abstract}

Index Terms-Affine systems, curvelets, denoising, shearlets, sparsity, video processing, wavelets.

\section{INTRODUCTION}

The shearlet representation, originally introduced in [1], [2], has emerged in recent years as one of the most effective frameworks for the analysis and processing of multidimensional data. This representation is part of a new class of multiscale methods introduced during the last 10 years with the goal to overcome the limitations of wavelets and other traditional methods through a framework which combines the standard multiscale decomposition and the ability to efficiently capture anisotropic features. Other notable such methods include the curvelets [3] and the contourlets [4]. Indeed, both curvelets and shearlets have been shown to form Parseval frames of $L^{2}\left(\mathbb{R}^{2}\right)$ which are (nearly) optimally sparse in the class of cartoon-like images, a standard model for images with edges [3], [5]. Specifically, if $f_{M}$ is the $M$ term approximation obtained by selecting the $M$ largest coefficients in the shearlet or curvelet expansion of a cartoon-like image $f$, then the approximation error satisfies the asymptotic estimate

$$
\left\|f-f_{M}^{S}\right\|_{2}^{2} \asymp M^{-2}(\log M)^{3}, \quad \text { as } M \rightarrow \infty .
$$

Copyright (c) 2010 IEEE. Personal use of this material is permitted However, permission to use this material for any other purposes must be obtained from the IEEE by sending a request to pubs-permissions @ieee.org.

P. S. Negi is with the Department of Mathematics, University of Houston, Houston, TX 77204, USA (e-mail:psnegi@math.uh.edu).

D. Labate is with the Department of Mathematics, University of Houston, Houston, TX 77204, USA (e-mail:dlabate@math.uh.edu).

EDICS: TEC-RST, TEC-MRS
Up to the log-like factor, this is the optimal approximation rate, in the sense that no other orthonormal systems or even frames can achieve a rate better than $M^{-2}$. By contrast, wavelet approximations can only achieve a rate $M^{-1}$ for functions in this class [3]. Concerning the topic of sparse approximations, it is important to recall that the relevance of this notion goes far beyond the applications to compression. In fact, constructing sparse representations for data in a certain class entails the intimate understanding of their true nature and structure, so that sparse representations also provide the most effective tool for tasks such as feature extraction and pattern recognition [6], [7].

Even though shearlets and curvelets share the same philosophy of combining multiscale and directional analysis and have similar sparsity properties, they rely on a rather different mathematical structure. In particular, the directionality of the shearlet systems is controlled through the use of shearing matrices rather than rotations, which are employed by curvelets. This offers the advantage of preserving the discrete integer lattice and enables a natural transition from the continuous to the discrete setting. The contourlets, on the other hand, are a purely discrete framework, with the emphasis in the numerical implementation rather than the continuous construction. The special properties of the shearlet approach have been successfully exploited in several imaging application. For example, the combination of multiscale and directional decomposition using shearing transformations is used to design powerful algorithms for image denoising in [7], [8]; the directional selectivity of the shearlet representation is exploited to derive very competitive algorithms for edge detection and analysis in [9]; the sparsity of the shearlet representation is used to derive a very effective algorithm for the regularized inversion of the Radon transform in [10]. We also recall that a recent construction of compactly supported shearlets appears to be promising in PDE's and other applications [11], [12].

While directional multiscale systems such as curvelets and shearlets have emerged several years ago, only very recently the analysis of sparse representations using these representations has been extended beyond dimension 2. This extension is of great interest since many applications from areas such as medical diagnostic, video surveillance and seismic imaging require to process $3 \mathrm{D}$ data sets, and sparse $3 \mathrm{D}$ representations are very useful for the design of improved algorithms for data analysis and processing.

Notice that the formal extension of the construction of multiscale directional systems from $2 \mathrm{D}$ to $3 \mathrm{D}$ is not the major challenge. In fact, 3D versions of curvelets have been introduced in [13], with the focus being on their discrete 

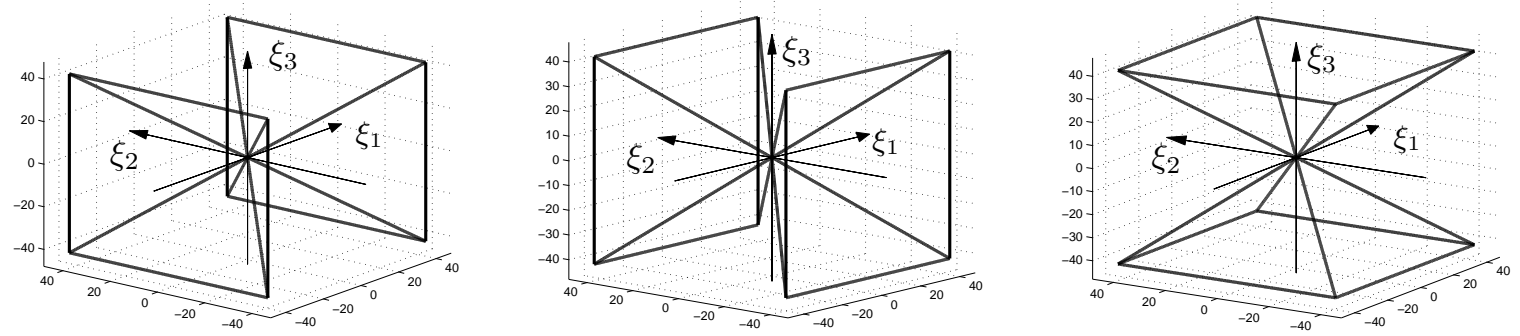

Fig. 1. From left to right, the figure illustrates the pyramidal regions $\mathcal{P}_{1}, \mathcal{P}_{2}$ and $\mathcal{P}_{3}$ in the frequency space $\widehat{\mathbb{R}}^{3}$.

implementations. Another discrete method is based on the system of surfacelets that were introduced as $3 \mathrm{D}$ extensions of contourlets in [14]. However, the analysis of the sparsity properties of curvelets or shearlets (or any other similar systems) in the 3D setting does not follow directly from the 2D argument. Only very recently [15], [16] it was shown by one of the authors in collaboration with $\mathrm{K}$. Guo that $3 \mathrm{D}$ shearlet representations exhibit essentially optimal approximation properties for piecewise smooth functions of 3 variables. Namely, for 3D functions $f$ which are smooth away from discontinuities along $C^{2}$ surfaces, it was shown that the $M$ term approximation $f_{M}^{S}$ obtained by selecting the $N$ largest coefficients in the 3D Parseval frame shearlet expansion of $f$ satisfies the asymptotic estimate

$$
\left\|f-f_{M}^{S}\right\|_{2}^{2} \asymp M^{-1}(\log M)^{2}, \quad \text { as } M \rightarrow \infty .
$$

Up to the logarithmic factor, this is the optimal decay rate and significantly outperforms wavelet approximations, which only yield a $M^{-1 / 2}$ rate for functions in this class.

It is useful to recall that optimal approximation properties for a large class of images can also be achieved using adaptive methods by using, for example, the bandelets [17] or the grouplets [18]. The shearlet approach, on the other hand, in non-adaptive. Remarkably, shearlets are able to achieve approximation properties which are essentially as good as an adaptive approach when dealing with the class of cartoon-like images.

The objective of the paper is to present a numerical implementation of the 3D Discrete Shearlet Transform which takes advantage of the sparsity properties of the corresponding continuous representation. To illustrate the performance of this new numerical algorithm, we consider a number of applications to problems of video denoising and enhancement. As it will become apparent from the results presented below, not only our video processing algorithm based on the 3D Discrete Shearlet Transform outperforms those based on the corresponding 2D Discrete Shearlet Transform (when applied "slice by slice"), but it is also extremely competitive against similar algorithms based on 3D curvelets and surfacelets.

\section{Shearlet Representations}

The shearlet approach provides a general method for the construction of function systems made up of functions ranging not only at various scales and locations, but also according to various orthogonal transformations controlled by shearing matrices.

In dimension $D=3$, a shearlet system is obtained by appropriately combining 3 systems of functions associated with the pyramidal regions

$$
\begin{aligned}
& \mathcal{P}_{1}=\left\{\left(\xi_{1}, \xi_{2}, \xi_{3}\right) \in \mathbb{R}^{3}:\left|\frac{\xi_{2}}{\xi_{1}}\right| \leq 1,\left|\frac{\xi_{3}}{\xi_{1}}\right| \leq 1\right\}, \\
& \mathcal{P}_{2}=\left\{\left(\xi_{1}, \xi_{2}, \xi_{3}\right) \in \mathbb{R}^{3}:\left|\frac{\xi_{1}}{\xi_{2}}\right|<1,\left|\frac{\xi_{3}}{\xi_{2}}\right| \leq 1\right\}, \\
& \mathcal{P}_{3}=\left\{\left(\xi_{1}, \xi_{2}, \xi_{3}\right) \in \mathbb{R}^{3}:\left|\frac{\xi_{1}}{\xi_{3}}\right|<1,\left|\frac{\xi_{2}}{\xi_{3}}\right|<1\right\},
\end{aligned}
$$

in which the Fourier space $\widehat{\mathbb{R}}^{3}$ is partitioned (see Fig. 1).

To define such systems, let $\phi$ be a $C^{\infty}$ univariate function such that $0 \leq \hat{\phi} \leq 1, \hat{\phi}=1$ on $\left[-\frac{1}{16}, \frac{1}{16}\right]$ and $\hat{\phi}=0$ outside the interval $\left[-\frac{1}{8}, \frac{1}{8}\right]$. That is, $\phi$ is the scaling function of a Meyer wavelet, rescaled so that its frequency support is contained the interval $\left[-\frac{1}{8}, \frac{1}{8}\right]$. For $\xi=\left(\xi_{1}, \xi_{2}, \xi_{3}\right) \in \widehat{\mathbb{R}}^{3}$, define

$$
\widehat{\Phi}(\xi)=\widehat{\Phi}\left(\xi_{1}, \xi_{2}, \xi_{3}\right)=\hat{\phi}\left(\xi_{1}\right) \hat{\phi}\left(\xi_{2}\right) \hat{\phi}\left(\xi_{3}\right)
$$

and let $W(\xi)=\sqrt{\widehat{\Phi}^{2}\left(2^{-2} \xi\right)-\widehat{\Phi}^{2}(\xi)}$. It follows that

$$
\widehat{\Phi}^{2}(\xi)+\sum_{j \geq 0} W^{2}\left(2^{-2 j} \xi\right)=1 \text { for } \xi \in \mathbb{R}^{3} .
$$

Notice that each function $W_{j}=W\left(2^{-2 j} \cdot\right), j \geq 0$, is supported inside the Cartesian corona

$$
\left[-2^{2 j-1}, 2^{2 j-1}\right]^{3} \backslash\left[-2^{2 j-4}, 2^{2 j-4}\right]^{3} \subset \widehat{\mathbb{R}}^{3},
$$

and the functions $W_{j}^{2}, j \geq 0$, produce a smooth tiling of $\widehat{\mathbb{R}}^{3}$. Next, let $V \in C^{\infty}(\mathbb{R})$ be such that $\operatorname{supp} V \subset[-1,1]$ and

$$
|V(u-1)|^{2}+|V(u)|^{2}+|V(u+1)|^{2}=1 \quad \text { for }|u| \leq 1 .
$$

In addition, we that $V(0)=1$ and that $V^{(n)}(0)=0$ for all $n \geq 1$. It was shown in [5] that there are several examples of functions satisfying these properties. It follows from equation (4) that, for any $j \geq 0$,

$$
\sum_{m=-2^{j}}^{2^{j}}\left|V\left(2^{j} u-m\right)\right|^{2}=1, \quad \text { for }|u| \leq 1 .
$$


For $d=1,2,3, \ell=\left(\ell_{1}, \ell_{2}\right) \in \mathbb{Z}^{2}$, the $3 \mathrm{D}$ shearlet systems associated with the pyramidal regions $\mathcal{P}_{d}$ are defined as the collections

$$
\left\{\psi_{j, \ell, k}^{(d)}: j \geq 0,-2^{j} \leq \ell_{1}, \ell_{2} \leq 2^{j}, k \in \mathbb{Z}^{3}\right\},
$$

where

$\hat{\psi}_{j, \ell, k}^{(d)}(\xi)=\left|\operatorname{det} A_{(d)}\right|^{-\frac{j}{2}} W\left(2^{-2 j} \xi\right) F_{(d)}\left(\xi A_{(d)}^{-j} B_{(d)}^{[-\ell]}\right) e^{2 \pi i \xi A_{(d)}^{-j} B_{(d)}^{[-\ell]} k}$,

$F_{(1)}\left(\xi_{1}, \xi_{2}, \xi_{3}\right)=V\left(\frac{\xi_{2}}{\xi_{1}}\right) V\left(\frac{\xi_{3}}{\xi_{1}}\right), \quad F_{(2)}\left(\xi_{1}, \xi_{2}, \xi_{3}\right) \stackrel{(7)}{=}$ $V\left(\frac{\xi_{1}}{\xi_{2}}\right) V\left(\frac{\xi_{3}}{\xi_{2}}\right), F_{(3)}\left(\xi_{1}, \xi_{2}, \xi_{3}\right)=V\left(\frac{\xi_{1}}{\xi_{3}}\right) V\left(\frac{\xi_{2}}{\xi_{3}}\right)$, the anisotropic dilation matrices $A_{(d)}$ are given by

$$
A_{(1)}=\left(\begin{array}{lll}
4 & 0 & 0 \\
0 & 2 & 0 \\
0 & 0 & 2
\end{array}\right), A_{(2)}=\left(\begin{array}{lll}
2 & 0 & 0 \\
0 & 4 & 0 \\
0 & 0 & 2
\end{array}\right), A_{(3)}=\left(\begin{array}{lll}
2 & 0 & 0 \\
0 & 2 & 0 \\
0 & 0 & 4
\end{array}\right),
$$

and the shear matrices are defined by

$$
B_{(1)}^{[\ell]}=\left(\begin{array}{ccc}
1 & \ell_{1} & \ell_{2} \\
0 & 1 & 0 \\
0 & 0 & 1
\end{array}\right), B_{(2)}^{[\ell]}=\left(\begin{array}{ccc}
1 & 0 & 0 \\
\ell_{1} & 1 & \ell_{2} \\
0 & 0 & 1
\end{array}\right), B_{(3)}^{[\ell]}=\left(\begin{array}{ccc}
1 & 0 & 0 \\
0 & 1 & 0 \\
\ell_{1} & \ell_{2} & 1
\end{array}\right) .
$$

Due to the assumptions on $W$ and $v$, the elements of the system of shearlets (6) are well localized and bandlimited. In particular, the shearlets $\hat{\psi}_{j, \ell, k}^{(1)}(\xi)$ can be written more explicitly as

$\hat{\psi}_{j, \ell, k}^{(1)}(\xi)=2^{-2 j} W\left(2^{-2 j} \xi\right) V\left(2^{j} \frac{\xi_{2}}{\xi_{1}}-\ell_{1}\right) V\left(2^{j} \frac{\xi_{3}}{\xi_{1}}-\ell_{2}\right) e^{2 \pi i \xi A_{(1)}^{-j} B_{(1)}^{[-\ell]} k}$

showing that their supports are contained inside the trapezoidal regions

$$
\begin{gathered}
\left\{\left(\xi_{1}, \xi_{2}, \xi_{3}\right): \xi_{1} \in\left[-2^{2 j-1},-2^{2 j-4}\right] \cup\left[2^{2 j-4}, 2^{2 j-1}\right],\right. \\
\left.\left|\frac{\xi_{2}}{\xi_{1}}-\ell_{1} 2^{-j}\right| \leq 2^{-j},\left|\frac{\xi_{3}}{\xi_{1}}-\ell_{2} 2^{-j}\right| \leq 2^{-j}\right\} .
\end{gathered}
$$

This expression shows that these support regions become increasingly more elongated at fine scales, due to the action of the anisotropic dilation matrices $A_{(1)}^{j}$, with the orientations of these regions controlled by the shearing parameters $\ell_{1}, \ell_{2}$. A typical support region is illustrated in Fig. 2. Similar properties hold for the elements associated with the regions $\mathcal{P}_{2}$ and $\mathcal{P}_{3}$.

A Parseval frame of shearlets for $L^{2}\left(\mathbb{R}^{3}\right)$ is obtained by using an appropriate combination of the systems of shearlets associated with the 3 pyramidal regions $\mathcal{P}_{d}, d=1,2,3$, together with a coarse scale system, which will take care of the low frequency region. In order to build such system in a way that all its elements are smooth in the Fourier domain, one has to appropriately define the elements of the shearlet systems overlapping the boundaries of the pyramidal regions $\mathcal{P}_{d}$ in the Fourier domain.

Hence, we define the $3 D$ shearlet systems for $L^{2}\left(\mathbb{R}^{3}\right)$ as the collections

$$
\begin{gathered}
\left\{\widetilde{\psi}_{-1, k}: k \in \mathbb{Z}^{3}\right\} \bigcup\left\{\widetilde{\psi}_{j, \ell, k}: j \geq 0, \ell_{1}, \ell_{2}= \pm 2^{j}, k \in \mathbb{Z}^{3}\right\} \\
\left\{\widetilde{\psi}_{j, \ell, k, d}: j \geq 0,\left|\ell_{1}\right|<2^{j},\left|\ell_{2}\right| \leq 2^{j}, k \in \mathbb{Z}^{3}, d=1,2,3\right\}
\end{gathered}
$$

consisting of:

- the coarse-scale shearlets $\left\{\widetilde{\psi}_{-1, k}=\Phi(\cdot-k): k \in \mathbb{Z}^{3}\right\}$, where $\Phi$ is given by (2);

- the interior shearlets $\left\{\widetilde{\psi}_{j, \ell, k, d}=\psi_{j, \ell, k}^{(d)}: j \geq 0,\left|\ell_{1}\right|\left|\ell_{2}\right|<\right.$ $\left.2^{j}, k \in \mathbb{Z}^{3}, d=1,2,3\right\}$, where $\psi_{j, \ell, k}^{(d)}$ are given by (7);

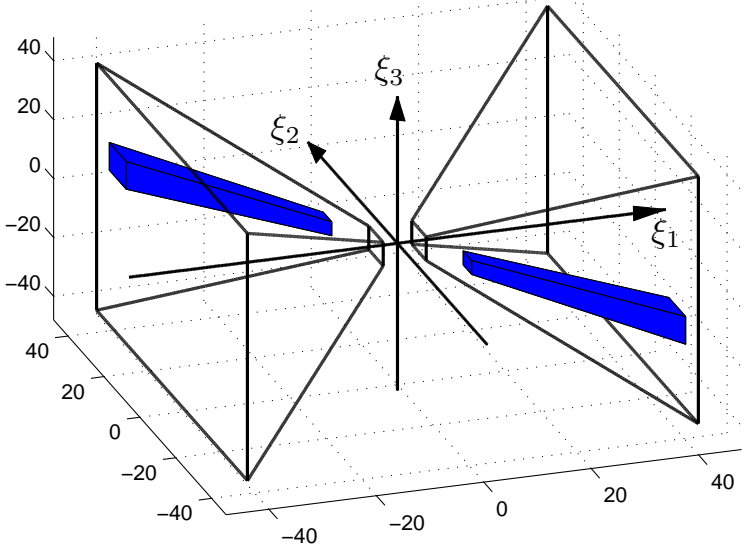

Fig. 2. Frequency support of a representative shearlet function $\psi_{j, \ell, k}$, inside the pyramidal region $\mathcal{P}_{1}$. The orientation of the support region is controlled by $\ell=\left(\ell_{1}, \ell_{2}\right)$; its shape is becoming more elongated as $j$ increases $(j=4$ in this plot).

- the boundary shearlets $\left\{\widetilde{\psi}_{j, \ell, k, d}: j \geq 0,\left|\ell_{1}\right| \leq 2^{j}, \ell_{2}=\right.$ $\left.\pm 2^{j}, k \in \mathbb{Z}^{3}, d=1,2,3\right\}$.

These boundary shearlets are obtained by joining together the functions $\psi_{j, \ell, k}^{(1)}, \psi_{j, \ell, k}^{(2)}$ and $\psi_{j, \ell, k}^{(3)}$, for $\ell_{1}= \pm 2^{j}$ or $\ell_{2}= \pm 2^{j}$, after they have been restricted to their respective pyramidal regions. For example (see [19] for all cases and additional detail), when $\ell_{1}= \pm 2^{j},\left|\ell_{2}\right|<2^{j}$, we define

$$
\begin{aligned}
& \left(\widetilde{\psi}_{j, \ell_{1}, \ell_{2}, k, 1}\right)^{\wedge}(\xi)
\end{aligned}
$$

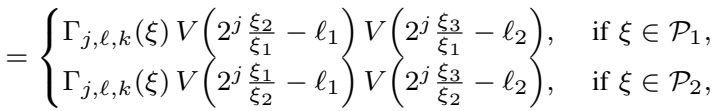

where

$$
\Gamma_{j, \ell, k}(\xi)=2^{-2 j} W\left(2^{-2 j} \xi\right) e^{2 \pi i \xi A_{(1)}^{-j} B_{(1)}^{\left[-\left(\ell_{1}, \ell_{2}\right)\right]} k} .
$$

When $\ell_{1}, \ell_{2}= \pm 2^{j}$, we define

$$
\begin{aligned}
& \left(\widetilde{\psi}_{j, \ell_{1}, \ell_{2}, k}\right)^{\wedge}(\xi) \\
& = \begin{cases}\Gamma_{j, \ell, k}(\xi) V\left(2^{j} \frac{\xi_{2}}{\xi_{1}}-\ell_{1}\right) V\left(2^{j} \frac{\xi_{3}}{\xi_{1}}-\ell_{2}\right), & \text { if } \xi \in \mathcal{P}_{1}, \\
\Gamma_{j, \ell, k}(\xi) V\left(2^{j} \frac{\xi_{1}}{\xi_{2}}-\ell_{1}\right) V\left(2^{j} \frac{\xi_{3}}{\xi_{2}}-\ell_{2}\right), & \text { if } \xi \in \mathcal{P}_{2}, \\
\Gamma_{j, \ell, k}(\xi) V\left(2^{j} \frac{\xi_{1}}{\xi_{3}}-\ell_{1}\right) V\left(2^{j} \frac{\xi_{2}}{\xi_{3}}-\ell_{2}\right), & \text { if } \xi \in \mathcal{P}_{3} .\end{cases}
\end{aligned}
$$

Notice that, thanks on the assumptions on $W$ and $V$, the piecewise defined boundary shearlet functions are smooth and compactly supported in the Fourier domain (see [19], [16] for additional detail). In addition, the system of shearlets (9) is a Parseval frame. To state this result, let us introduce the following compact notation to write the 3D shearlet system (9) as

$$
\left\{\widetilde{\psi}_{\mu}, \mu \in \mathcal{M}\right\},
$$

where $\mathcal{M}=\mathcal{M}_{C} \cup \mathcal{M}_{I} \cup \mathcal{M}_{B}$ are the indices associated with the coarse-scale shearlets, the interior shearlets and the boundary shearlets given by

$$
\text { - } \mathcal{M}_{C}=\left\{\mu=(j, k): j=-1, k \in \mathbb{Z}^{3}\right\} \text {; }
$$


- $\mathcal{M}_{I}=\left\{\mu=\left(j, \ell_{1}, \ell_{2}, k, d\right): j \geq 0,\left|\ell_{1}\right|,\left|\ell_{2}\right|<2^{j}, k \in\right.$ $\left.\mathbb{Z}^{3}, d=1,2,3\right\}$

- $\mathcal{M}_{B}=\left\{\mu=\left(j, \ell_{1}, \ell_{2}, k, d\right): j \geq 0,\left|\ell_{1}\right| \leq 2^{j}, \ell_{2} \pm\right.$ $\left.2^{j}, k \in \mathbb{Z}^{3}, d=1,2,3\right\}$.

Hence we have the following result whose proof is found in [19]:

Theorem 1: The $3 D$ system of shearlets (12) is a Parseval frame of $L^{2}\left(\mathbb{R}^{3}\right)$. That is, for any $f \in L^{2}\left(\mathbb{R}^{3}\right)$,

$$
\sum_{\mu \in \mathcal{M}}\left|\left\langle f, \widetilde{\psi}_{\mu}\right\rangle\right|^{2}=\|f\|^{2} .
$$

The mapping from $f \in L^{2}\left(\mathbb{R}^{3}\right)$ into the elements $\left\langle f, \widetilde{\psi}_{\mu}\right\rangle$, $\mu \in \mathcal{M}$, is called the $3 D$ shearlet transform.

As mentioned above, it is proved in [15], [16] that the 3D Parseval frame of shearlets $\left\{\widetilde{\psi}_{\mu}, \mu \in \mathcal{M}\right\}$ achieves the essentially optimal approximation rate (1) for functions of 3 variables which are $C^{2}$ regular away from discontinuities along $C^{2}$ surfaces.

\section{3D Discrete Shearlet Transform (3D DShT)}

In this section, we present a digital implementation of the 3D shearlet transform introduced above. Following essentially the same architecture as the algorithm of the 2D Discrete Shearlet Transform in [8], this new implementation can be described as the cascade of a multiscale decomposition, based on a version of the Laplacian pyramid filter, followed by a stage of directional filtering. The main novelty of the 3D approach consists in the design of the directional filtering stage, which attempts to faithfully reproduce the frequency decomposition provided by the corresponding mathematical transform by using a method based on the pseudo-spherical Fourier transform.

Let us start by expressing the elements of the shearlet system in a form that is more convenient for deriving an algorithmic implementation of the shearlet transform. For $\xi=\left(\xi_{1}, \xi_{2}, \xi_{3}\right)$ in $\widehat{\mathbb{R}}^{3}, j \geq 0$, and $-2^{j} \leq \ell_{1}, \ell_{2} \leq 2^{j}$, we define the directional windowing functions

$$
\begin{gathered}
U_{j, \ell}^{(1)}(\xi)= \\
\begin{cases}V\left(2^{j} \frac{\xi_{2}}{\xi_{1}}-\ell_{1}\right) V\left(2^{j} \frac{\xi_{3}}{\xi_{1}}-\ell_{2}\right) & \text { if }\left|\ell_{1}\right|,\left|\ell_{2}\right|<2^{j} \\
V\left(2^{j} \frac{\xi_{2}}{\xi_{1}}-\ell_{1}\right) V\left(2^{j} \xi_{3}\right. & \left.\ell_{2}\right) \mathcal{X}_{\mathcal{P}_{1}}(\xi) \\
+V\left(2^{j} \frac{\xi_{1}}{\xi_{2}}-\ell_{1}\right) V\left(2^{j} \frac{\xi_{3}}{\xi_{2}}-\ell_{2}\right) \mathcal{X}_{\mathcal{P}_{2}}(\xi) & \text { if } \ell_{1}= \pm 2^{j},\left|\ell_{2}\right|<2^{j} \\
V\left(2^{j} \xi_{2}\right. & \left.\xi_{1}-\ell_{1}\right) V\left(2^{j} \xi_{3}-\ell_{2}\right) \mathcal{X}_{\mathcal{P}_{1}}(\xi) \\
+V\left(2^{j} \frac{\xi_{1}}{\xi_{1}}-\ell_{1}\right) V\left(2^{j} \frac{\xi_{3}}{\xi_{2}}-\ell_{2}\right) \mathcal{X}_{\mathcal{P}_{2}}(\xi) & \\
+V\left(2^{j} \frac{\xi_{1}}{\xi_{3}}-\ell_{1}\right) V\left(2^{j} \frac{\xi_{2}}{\xi_{3}}-\ell_{2}\right) \mathcal{X}_{\mathcal{P}_{3}}(\xi) & \text { if } \ell_{1}, \ell_{2}= \pm 2^{j}\end{cases}
\end{gathered}
$$

Notice that only the elements $U_{j, \ell}^{(1)}$ with indices $\left|\ell_{1}\right|,\left|\ell_{2}\right|<$ $2^{j}$ are strictly contained inside the region $\mathcal{P}_{1}$; the elements with indices $\ell_{1}= \pm 2^{j}$ or $\ell_{2}= \pm 2^{j}$ are supported across $\mathcal{P}_{1}$ and some other pyramidal region. However, it is convenient to associate this family of functions with the index 1 . We define the functions $U_{j, \ell}^{(2)}$ and $U_{j, \ell}^{(3)}$ associated with the pyramidal regions $\mathcal{P}_{2}$ and $\mathcal{P}_{3}$ in a similar way ${ }^{1}$. Using this notation, we can write each element of the $3 \mathrm{D}$ shearlet system as

$$
\hat{\psi}_{j, \ell, k}^{(d)}=2^{-2 j} W\left(2^{-2 j} \xi\right) U_{j, \ell}^{(d)}(\xi) e^{-2 \pi i \xi A_{(d)}^{-j} B_{d}^{[-\ell]} k} .
$$

${ }^{1}$ Notice however that they do not contain the boundary term for $\ell_{1}, \ell_{2}=$ $\pm 2^{j}$, which only needs to be included once.
It follows from the properties of the shearlet construction that

$$
\sum_{d=1}^{3} \sum_{j \geq 0} \sum_{\ell_{1}=-2^{j}}^{2^{j}} \sum_{\ell_{2}=-2^{j}}^{2^{j}} \mid W\left(\left.2^{-2 j}(\xi)\right|^{2}\left|U_{j, \ell}^{(d)}(\xi)\right|^{2}=1,\right.
$$

for $\left|\xi_{1}\right|,\left|\xi_{2}\right|,\left|\xi_{3}\right| \geq \frac{1}{8}$. The (fine scale) 3D shearlet transform of $f \in L\left(\mathbb{R}^{3}\right)$ can be expressed as the mapping from $f$ into the shearlet coefficients

$$
\left\langle f, \psi_{j, \ell, k}^{(d)}\right\rangle=\int_{\mathbb{R}^{3}} \hat{f}(\xi) W\left(2^{-2 j} \xi\right) U_{j, \ell}^{(d)}(\xi) e^{2 \pi i \xi A_{(d)}^{-j} B_{(d)}^{[-\ell]} k} d \xi,(14)
$$

where $j \geq 0, \ell=\left(\ell_{1}, \ell_{2}\right)$ with $\left|\ell_{1}\right|,\left|\ell_{2}\right| \leq 2^{j}, k \in \mathbb{Z}^{3}$ and $d=1,2,3$.

This expression shows that the shearlet transform of $f$, for $j, \ell, k$ and $d$ fixed, can be computed using the following steps:

1) In the frequency domain, compute the $j$-th subband decomposition of $f$ as $\hat{f}_{j}(\xi)=\hat{f}(\xi) W\left(2^{-2 j} \xi\right)$.

2) Next (still in the frequency domain), compute the $(j, \ell, d)$-th directional subband decomposition of $f$ as $\hat{f}_{j, \ell, d}(\xi)=\hat{f}_{j}(\xi) U_{j, \ell}^{(d)}(\xi)$.

3) Compute the inverse Fourier transform. This step can be represented as a convolution of the $j$-th subband decomposition of $f$ and the directional filter $\check{U}_{j, \ell}^{(d)}$, that is, $\left\langle f, \psi_{j, \ell, k}^{(d)}\right\rangle=f_{j} * \check{U}_{j, \ell}^{(d)}\left(A_{d}^{-j} B_{d}^{-\ell} k\right)$.

Hence, the shearlet transform of $f$ can be described as a cascade of subband decomposition and directional filtering stage.

\section{A. 3D DShT Algorithm}

The new numerical algorithm for computing the digital values of the 3D shearlet transform, which is called $3 D D S h T$ algorithm, will follow closely the 3 steps indicated above.

Before describing the numerical algorithm, let us recall that a digital 3D function $f$ is an element of $\ell^{2}\left(\mathbb{Z}_{N}^{3}\right)$, where $N \in \mathbb{N}$, that is, it consists of a finite array of values $\left\{f\left[n_{1}, n_{2}, n_{3}\right]\right.$ : $\left.n_{1}, n_{2}, n_{2}=0,1,2, \ldots, N-1\right\}$. Here and in the following, we adopt the convention that a bracket $[\cdot, \cdot, \cdot]$ denotes an array of indices whereas the standard parenthesis $(\cdot, \cdot, \cdot)$ denotes a function evaluation. Given a $3 D$ digital function $f \in \ell^{2}\left(\mathbb{Z}_{N}^{3}\right)$, its Discrete Fourier Transform is given by:

$\hat{f}\left[k_{1}, k_{2}, k_{3}\right]=N^{-\frac{3}{2}} \sum_{n_{1}, n_{2}, n_{3}=0}^{N-1} f\left[n_{1}, n_{2}, n_{3}\right] e^{\left(-2 \pi i\left(\frac{n_{1}}{N} k_{1}+\frac{n_{2}}{N} k_{2}+\frac{n_{3}}{N} k_{3}\right)\right)}$

for $\frac{N}{2} \leq k_{1}, k_{2}, k_{3}<\frac{N}{2}$. We shall interpret the numbers $\hat{f}\left[k_{1}, k_{2}, k_{3}\right]$ as samples $\hat{f}\left[k_{1}, k_{2}, k_{3}\right]=\hat{f}\left(k_{1}, k_{2}, k_{3}\right)$ from the trigonometric polynomial

$\hat{f}\left(\xi_{1}, \xi_{2}, \xi_{3}\right)=N^{-\frac{3}{2}} \sum_{n_{1}, n_{2}, n_{3}=0}^{N-1} f\left[n_{1}, n_{2}, n_{3}\right] e^{\left(-2 \pi i\left(\frac{n_{1}}{N} \xi_{1}+\frac{n_{2}}{N} \xi_{2}+\frac{n_{3}}{N} \xi_{3}\right)\right)}$.

We can now proceed with the description of the implementation of the 3D DShT algorithm.

First, to calculate $\hat{f}_{j}(\xi)$ in the digital domain, we perform the computation in the DFT domain as the product of the DFT of $f$ and the DFT of the filters $w_{j}$ corresponding to the bandpass functions $W\left(2^{-2 j}\right.$.). This step can be implemented using the Laplacian pyramid algorithm [20], which results in the decomposition of the input signal $f \in \ell^{2}\left(\mathbb{Z}_{N}^{3}\right)$ into a low-pass and high-pass components. After extensive testing, 
we found that a very satisfactory performance is achieved using the modified version of the Laplacian pyramid algorithm developed in [14]. For the first level of the decomposition, this algorithm downsamples the low-pass output by a non-integer factor of 1.5 (upsampling by 2 followed by downsampling by 3) along each dimension; the high-pass output is not downsampled. In the subsequent decomposition stages, the low-pass output is downsampled by 2 along each dimension and the high-pass output is not downsampled. Although the fractional sampling factor in the first stage makes the algorithm slightly more redundant than the traditional Laplacian pyramid, it was found that the added redundancy is very useful in reducing the frequency domain aliasing (see [14] for more detail).

Next, one possible approach for computing the directional components $\hat{f}_{j, \ell, d}$ of $\hat{f}$ consists in resampling the $j$-th subband component of $f$ into a pseudo-spherical grid and applying a two-dimensional band-pass filter. Even though this is not the approach we will use for our numerical experiments, the method that we will use is conceptually derived from this one.

Recall that the pseudo-spherical grid is the 3D extension of the 2D pseudo-polar grid and is parametrized by the planes going through the origin and their slopes. That is, the pseudospherical coordinates $(u, v, w) \in \mathbb{R}^{3}$ are given by

$$
(u, v, w)= \begin{cases}\left(\xi_{1}, \frac{\xi_{2}}{\xi_{1}}, \frac{\xi_{3}}{\xi_{1}}\right) & \text { if }\left(\xi_{1}, \xi_{2}, \xi_{3}\right) \in \mathcal{D}_{C_{1}}, \\ \left(\xi_{2}, \frac{\xi_{1}}{\xi_{2}}, \frac{\xi_{3}}{\xi_{2}}\right) & \text { if }\left(\xi_{1}, \xi_{2}, \xi_{3}\right) \in \mathcal{D}_{C_{2}}, \\ \left(\xi_{3}, \frac{\xi_{1}}{\xi_{3}}, \frac{\xi_{2}}{\xi_{3}}\right) & \text { if }\left(\xi_{1}, \xi_{2}, \xi_{3}\right) \in \mathcal{D}_{C_{3}} .\end{cases}
$$

Using this change of variables, it follows that $\hat{f}_{j, \ell, d}(\xi)$ can be written as

$$
\hat{g}_{j}(u, v, w) U^{(d)}\left(u, 2^{j} v-\ell_{1}, 2^{j} w-\ell_{2}\right),
$$

where $\hat{g}_{j}(u, v, w)$ is the function $\hat{f}_{j}(\xi)$, after the change of variables, and $U^{(d)}=U_{0,0}^{(d)}$. Notice that $U^{(d)}$ does not depend on $u$. For example, when $d=1$, the expression (15) can be written as

$$
\hat{g}_{j}(u, v, w) V\left(2^{j} v-\ell_{1}\right) V\left(2^{j} w-\ell_{2}\right),
$$

showing that the different directional components of $\hat{f}_{j}$ are obtained by simply translating the window function $V$ in the pseudo-spherical domain. In fact, this is a direct consequence of using shearing matrices to control orientations and is its main advantage with respect to rotations. As a result, the discrete samples $g_{j}\left[n_{1}, n_{2}, n_{3}\right]=g_{j}\left(n_{1}, n_{2}, n_{3}\right)$ are the values of the DFT of $f_{j}\left[n_{1}, n_{2}, n_{3}\right]$ on the pseudo-spherical grid and they can be computed by direct reassignment or by adapting the Pseudo-polar DFT algorithm [21], [22] to the 3D setting. The 3D Pseudo-polar DFT evaluates the Fourier transform of the data on the Pseudo-polar grid and is formally defined as

$$
\begin{aligned}
& \hat{P}_{1}(f)(k, l, j):=\hat{f}\left(k,-\frac{2 l}{N} k,-2 \frac{2 j}{N} k\right), \\
& \hat{P}_{2}(f)(k, l, j):=\hat{f}\left(-\frac{2 l}{N} k, k,-2 \frac{2 j}{N} k\right), \\
& \hat{P}_{3}(f)(k, l, j):=\hat{f}\left(-\frac{2 l}{N} k,-2 \frac{2 j}{N} k, k\right),
\end{aligned}
$$

for $k=-\frac{2 N}{2}, \cdots, \frac{2 N}{2}$ and $l, k=-\frac{N}{2}, \cdots, \frac{N}{2}$.
Let $\left\{u_{j, \ell_{1}, \ell_{2}}^{(d)}\left[n_{2}, n_{3}\right]: n_{2}, n_{3} \in \mathbb{Z}\right\}$ be the sequence whose DFT gives the discrete samples of the window functions $U^{(d)}\left(2^{j} v-\ell_{1}, 2^{j} w-\ell_{2}\right)$. For example, when $d=1$, we have that $u_{j, \ell_{1}, \ell_{2}}^{(1)}\left[k_{2}, k_{3}\right]=V\left(2^{j} k_{2}-\ell_{1}\right) V\left(2^{j} k_{3}-\ell_{2}\right)$. Then, for fixed $k_{1} \in \mathbb{Z}$, we have

$$
\begin{aligned}
& \mathcal{F}_{2}\left(\mathcal{F}_{2}^{-1}\left(\hat{g}_{j}\right) * \check{u}_{j, \ell_{1}, \ell_{2}}^{(d)}\left[n_{2}, n_{3}\right]\right)\left[k_{1}, k_{2}, k_{3}\right] \\
& =\hat{g}_{j}\left[k_{1}, k_{2}, k_{3}\right] u_{j, \ell_{1}, \ell_{2}}^{(d)}\left[k_{2}, k_{3}\right]
\end{aligned}
$$

where $\mathcal{F}_{2}$ is the two dimensional DFT, defined as

$$
\mathcal{F}_{2}(f)\left[k_{2}, k_{3}\right]=\frac{1}{N} \sum_{n_{2}, n_{3}=0}^{N-1} f\left[n_{2}, n_{3}\right] e^{\left(-2 \pi i\left(\frac{n_{2}}{N} k_{2}+\frac{n_{3}}{N} k_{3}\right)\right)},
$$

for $-\frac{N}{2} \leq k_{2}, k_{3}<\frac{N}{2}$. Equation (16) gives the algorithmic procedure for computing the discrete samples of the right hand side of (15). That is, the 3D shearlet coefficients (14) can be calculated from equation (16) by computing the inverse pseudo-spherical DFT by directly re-assembling the Cartesian sampled values and applying the inverse 3-dimensional DFT.

In fact, the last observation suggests an alternative approach for computing the directional components $\hat{f}_{j, \ell, d}$ of $f$. This approach was found to perform better and it was used to produce the numerical results below. The main idea consists in mapping the filters from the pseudo-spherical domain back into the Cartesian domain and then perform a convolution with band-passed data, similar to one of the methods used for the 2D setting in [8]. Specifically if $\phi_{P}$ is the mapping from Cartesian domain into the pseudo-spherical domain then the 3D shearlet coefficients in the Fourier domain can be expressed as

$$
\phi_{P}^{-1}\left(\hat{g}_{j}\left[k_{1}, k_{2}, k_{3}\right] u_{j, \ell_{1}, \ell_{2}}^{(d)}\left[k_{2}, k_{3}\right]\right) .
$$

Following the approach in [8], this can be expressed as

$$
\phi_{P}^{-1}\left(\hat{g}_{j}\left[k_{1}, k_{2}, k_{3}\right]\right) \phi_{P}^{-1}\left(\hat{\delta}_{P}\left[k_{1}, k_{2}, k_{3}\right] u_{j, \ell_{1}, \ell_{2}}^{(d)}\left[k_{2}, k_{3}\right]\right),
$$

where $\hat{\delta}_{P}$ is the DFT of the (discrete) delta distribution in the pseudo-spherical grid. Thus the 3D discrete shearlet coefficients in the Fourier domain can be expressed as

$$
\hat{f}_{j}\left[k_{1}, k_{2}, k_{3}\right] \hat{h}_{j, \ell_{1}, \ell_{2}}^{(d)}\left[k_{1}, k_{2}, k_{3}\right] \text {, }
$$

where

$$
\hat{h}_{j, \ell_{1}, \ell_{2}}^{(d)}\left[k_{1}, k_{2}, k_{3}\right]=\phi_{P}^{-1}\left(\hat{\delta}_{P}\left[k_{1}, k_{2}, k_{3}\right] u_{j, \ell_{1}, \ell_{2}}^{(d)}\left[k_{2}, k_{3}\right]\right) .
$$

Notice that the new filters $h_{j, \ell_{1}, \ell_{2}}^{(d)}$ are not obtained by a simple change of variables, but by applying a resampling which converts the pseudo-spherical grid to a Cartesian grid. This resampling is done using a linear map where possibly several points from the polar grid are mapped to the same point on the rectangular grid. Although these filters are not compactly supported, they can be implemented with a matrix representation that is smaller than the size of the data $f$, hence allowing to implement the computation of the 3D DShT using a convolution in space domain. One benefit of this approach is that one does not need to resample the DFT of the data into a pseudo-spherical grid, as required using the first method.

Since the computational effort is essentially determined by the FFT which is used to transform data and compute 
convolutions, it follows that the 3D DShT algorithm runs in $O\left(N^{3} \log (N)\right)$ operations.

\section{B. Implementation issues.}

In principle, for the implementation of the 3D DShT algorithm one can choose any collection of filters $U_{j, \ell}^{(d)}$ as long as the tiling condition (13) is satisfied. The simplest solution is to choose functions $U_{j, \ell}^{(d)}$ which are characteristic functions of appropriate trapezoidal regions in the frequency domain, but this type of filters are poorly localized in space domain. To be faithful to the continuous construction and also to ensure well localized filters in the space domain, our implementation uses filters of Meyer type. A similar choice was also found effective in [8] for the 2D setting. As mentioned above, by taking the inverse DFT, it is possible to implement these filters using matrix representations of size $L^{3}$ with $L \ll N$, where $N^{3}$ is the data size. In the numerical experiment considered below, we have chosen $L=24$, which was found to be a very good compromise between localization and computation times. Finally, for the number of directional bands, our algorithm allows us to choose a different number of directional bands in each pyramidal region. The theory prescribes to choose a number $n$ of directional bands which, in each pyramidal region, grows like $2^{2 j}$, hence giving $n=4,16,64, \ldots$ directional bands, as the scale is becoming finer. As will discuss below, we found it convenient to slightly modify this canonical choice in the video denoising applications.

As a first illustration of the new 3D shearlet decomposition, we have run the 3D DShT algorithm using the Tempete video, of size $192^{3}$ voxels. Fig. 3 shows some representative 2D frames reconstructed from the 3-level 3D DShT decomposition of the Tempete video sequence. In particular, the figure shows a frame reconstructed from the approximation levels and some frames reconstructed from some representative directional subbands. The reconstruction from the directional subbands reported in this figure indicates that the shearlet decomposition is very sensitive to directional features.

\section{Correlation With Theory}

The numerical implementation of the 3D shearlet transform attempts to faithfully reproduce the frequency footprint associated with the 3D shearlet decomposition. Hence, it is natural to ask how does this numerical implementation behave with respect to the theoretical estimate (1).

To demonstrate that the approximation properties predicted by the theory are reflected in the approximation properties of the digital implementation, we have run some numerical experiments using a piece-wise constant radial function $f$ with jump discontinuities of the form

$$
f(x, y, z)=c_{i} \text { if } r_{i} \leq x^{2}+y^{2}+z^{2}<r_{i+1},
$$

for given vectors $c=\left(c_{i}\right)$ in $\mathbb{R}^{n}, r=\left(r_{i}\right)$ in $\mathbb{R}^{n+1}$. For example, by choosing $r=$ $(1,10,18,27,36,44,53,62,70,79,87,96) \quad$ and $c=(50,0,120,35,100,180,5,200,20,220,1,240)$, we found that the error $\left\|f-f_{M}^{S}\right\|$ decays like $M^{-0.6192}$ for our test image, as compared to a theoretical rate which

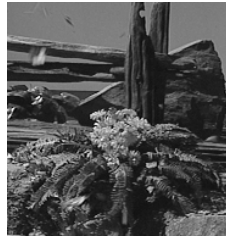

Original

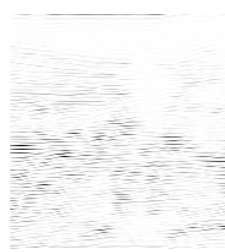

1st level Detail $\left(\ell_{1}=\ell_{2}=4\right) \quad$ 2nd level Detail $\left(\ell_{1}=\ell_{2}=2\right)$

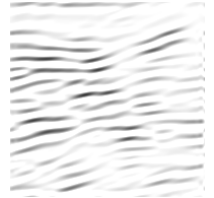

3rd level Detail $\left(\ell_{1}=\ell_{2}=2\right)$

Fig. 3. 3D DShT Decomposition of Tempete movie. The figure illustrates some representative $2 \mathrm{D}$ frames reconstructed from the 3D DShT decomposition of the movie. All detail frames are extracted from directional subbands contained in the pyramidal region $D_{\mathcal{C}_{1}}$. Detail frames, which show highly directional features, are shown in inverted gray scale.

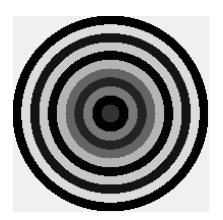

(a)

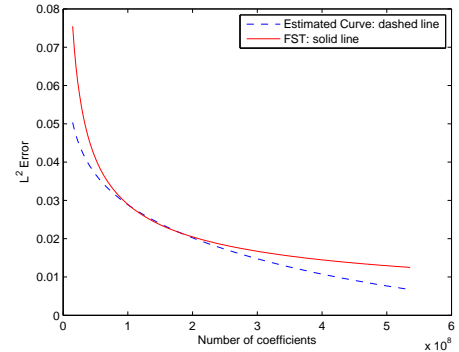

(b)
Fig. 4. Analysis of the nonlinear approximation error using the $3 D$ DShT algorithm. (a) Cross section of the piecewise constant radial function $f\left(\right.$ on $\left.\mathbb{R}^{3}\right)$. (b) Approximation error $\left\|f-f_{M}\right\|_{2}$.

is of the order $(\log M) M^{-0.5}$. Here $f_{M}$ is the nonlinear approximation of $f$ obtained using the $M$ largest shearlet coefficient in its 3D DShT expansion. The results of this test are plotted in Fig. 4 showing the nonlinear approximation error $\left\|f-f_{M}^{S}\right\|$ and comparing this plot to the theoretical curve $(\log M) M^{-0.5}$.

\section{NUMERICAL EXPERIMENTS}

As in the 2D setting, the ability of the 3D shearlet transform to deal with geometric information efficiently and its sparsity properties have the potential to produce significant improvement in many 3D data processing applications. As examples of these applications, we have developed algorithms for video 
denoising and enhancement which are based on the new 3D Discrete Shearlet Tranform presented above.

\section{A. Video Denoising}

The denoising of video is highly desirable for enhanced perceptibility, better compression and pattern recognition applications. While noise can have different distributions like Poisson, Laplacian or Gaussian distribution, we only considered the situation of zero-mean additive white Gaussian noise, which offers a good model for many practical situations. Hence, we assume that, for a given video $f$, we observe

$$
y=f+n,
$$

where $n$ is Gaussian white noise with zero mean and standard deviation $\sigma$.

It is well known that the ability to sparsely represent data is very useful in decorrelating the signal from the noise. This notion has been precisely formalized in the classical wavelet shrinkage approach by Donoho and Johnstone [23], [24], which has lead to many successful denoising algorithms. In the following, we adapt this idea to design a simple video denoising routine based on hard thresholding. That is, in our approach, we attempt to recover the video $f$ from the observed data $y$ as follows.

1) We compute the $3 \mathrm{D}$ shearlet decomposition of $y$ as $y=$ $\sum_{\mu}\left\langle y, \widetilde{\psi}_{\mu}\right\rangle \widetilde{\psi}_{\mu}$

2) We set to zero the coefficients $c_{\mu}(y)=\left\langle y, \widetilde{\psi}_{\mu}\right\rangle$ such that $\left|c_{\mu}(y)\right|<T$, where $T$ depends on the noise level.

3) We obtain an approximation $\tilde{f}$ of $f$ as $\tilde{f}=$ $\sum_{\mu} c_{\mu}^{*}(y) \widetilde{\psi}_{\mu}$, where

$$
c_{\mu}^{*}(y)= \begin{cases}c_{\mu}(y) & \text { if }\left|c_{\mu}(y)\right| \geq T \\ 0 & \text { otherwise. }\end{cases}
$$

For the choice of the threshold parameter, we adopt the same criterion which was found successful in the 2D setting, based on the classical BayesShrink method [25]. This consists in choosing

$$
T_{j, \ell}=\frac{\sigma^{2}}{\sigma_{j, \ell}},
$$

where $\sigma_{j, \ell}$ is the standard deviation of the shearlet coefficients in the $(j, \ell)$-th subband. Although hard thresholding is a rather crude form of thresholding and more sophisticated methods are available, still this method is a good indication of the potential of a transform in denoising applications. Also notice that hard thresholding performs better when dealing with data where it is important to preserve edges and sharp discontinuities (cf. [10], [26]).

For the 3D discrete shearlet decomposition, in all our tests we have applied a 3-level decomposition according to the algorithm described above. For the number of directional bands, we have chosen $n=16,16,64$ (from the coarsest to the fines level) in each of the pyramidal region. Even though this does not exactly respect the rule canonical choice ( $n=4,16,64$ ) prescribed by the continuous model, we found that increasing the number of directional subbands at the coarser level produces some improvement in the denoising

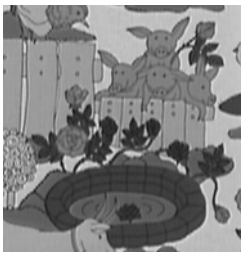

Original frame

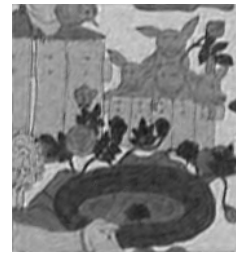

3D SHEAR

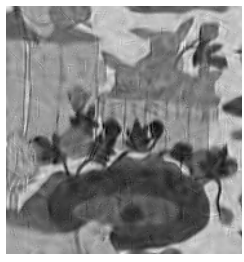

2D SHEAR

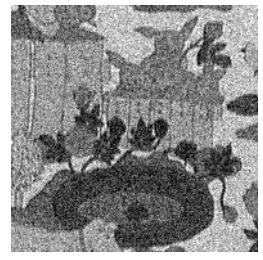

Noisy frame

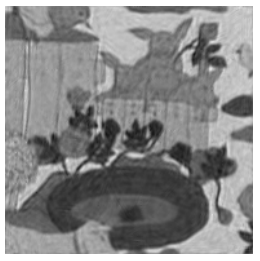

SURF

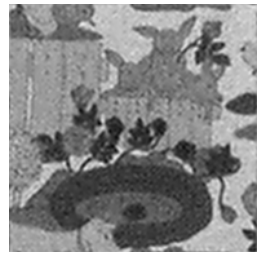

DWT
Fig. 5. Video Denoising of Mobile Video Sequence. The figure compares the denoising performance of the denoising algorithm based on the 3D DShT, denoted as 3DSHEAR, on a representative frame of the video sequence Mobile against various video denoising routines. Starting from the top left: original frame, noisy frame (PSNR=18.62 dB, corresponding to $\sigma=30$ ), denoised frame using 3DSHEAR (PSNR=28.68 dB), SURF (PSNR=28.39 $\mathrm{dB}), 2 \mathrm{DSHEAR}(\mathrm{PSNR}=25.97 \mathrm{~dB})$ and DWT $(\mathrm{PSNR}=24.93 \mathrm{~dB})$.

performance. Recall that, as indicated above, in our numerical implementation, downsampling occurs only at the bandpass level, and there is no anisotropic down-sampling. Thus, the numerical implementation of the 3D DShT which we found most effective in the denoising algorithm is highly redundant. Specifically, for data set of size $N^{3}$, a 3-level 3D DShT decomposition produces $3 *\left(64 * N^{3}+16 *\left(\frac{2}{3} N\right)^{3}+16 *\left(\frac{2}{6} N\right)^{3}\right)+$ $\left(\frac{2}{6} N\right)^{3} \approx 208 * N^{3}$ coefficients. As we will see below (Table II), this requires a higher computational cost than less redundant algorithms.

The 3D shearlet-based thresholding algorithm was tested on 3 video sequences, called mobile, coastguard and tempete, for various values of the standard deviation $\sigma$ of the noise (values $\sigma=30,40,50$ were considered). All these video sequences, which have been resized to $192 \times 192 \times 192$, can be uploaded from the website http://www. cipr.rpi.edu. For a baseline comparison, we tested the performance of the shearlet-based denoising algorithm (denoted by 3DSHEAR) against the following state-of-the-art algorithms: 3D Curvelets (denoted by 3DCURV, cf. [13]), Undecimated Discrete Wavelet Transform (denoted by UDWT, based on symlet of length 16), Dual Tree Wavelet Transform (denoted by DTWT, cf. [27]) and Surfacelets (denoted by SURF, cf. [14]). We also compared against the 2D discrete shearlet transform (denoted by 2DSHEAR), which was applied frame by frame, 
in order to illustrate the benefit of using a 3D transform, rather than a $2 \mathrm{D}$ transform acting on each frame.

As a performance measure, we used the standard peak signal-to-noise ratio (PSNR), measured in decibel(dB), which is defined by

$$
\operatorname{PSNR}=20 \log _{10} \frac{255 N}{\|f-\tilde{f}\|_{F}},
$$

where $\|\cdot\|_{F}$ is the Frobenius norm and $f$ is an array of size $N \times N \times N$.

The performance of the shearlet-based denoising algorithm 3DSHEAR relative to the other algorithms is shown in Table I, with the numbers in bold indicating the best performance. Notice that performance values for the algorithms 3DCURV, UDWT and DTWT are taken from [14].

Table I: Video denoising performance using different video sequences.

\begin{tabular}{|c|c|c|c|c|c|c|c|c|c|}
\hline PSNR $(\mathrm{dB})$ & \multicolumn{3}{|c|}{ Mobile } & \multicolumn{3}{c|}{ Coastguard } & \multicolumn{3}{c|}{ Tempete } \\
\hline \hline Noise $\sigma$ & 30 & 40 & 50 & 30 & 40 & 50 & 30 & 40 & 50 \\
\hline 3DCURV & 23.54 & 23.19 & 22.86 & 25.05 & 24.64 & 24.29 & & & \\
\hline UDWT & 24.02 & 22.99 & 22.23 & 25.95 & 24.95 & 24.2 & & & \\
\hline DTWT & 24.56 & 23.43 & 22.58 & 26.06 & 25.01 & 24.22 & & & \\
\hline SURF & 28.39 & $\mathbf{2 7 . 1 8}$ & $\mathbf{2 6 . 2 7}$ & 26.82 & 25.87 & $\mathbf{2 5 . 1 5}$ & 24.2 & 23.26 & 22.61 \\
\hline 3DSHEAR & $\mathbf{2 8 . 6 8}$ & 27.15 & 25.97 & $\mathbf{2 7 . 3 6}$ & $\mathbf{2 6 . 1 0}$ & 25.12 & $\mathbf{2 5 . 2 4}$ & $\mathbf{2 3 . 9 7}$ & $\mathbf{2 2 . 8 1}$ \\
\hline 2DSHEAR & 25.97 & 24.40 & 23.20 & 25.20 & 23.82 & 22.74 & 22.89 & 21.63 & 20.75 \\
\hline DWT & 24.93 & 23.94 & 23.03 & 24.34 & 23.44 & 22.57 & 22.09 & 21.5 & 20.92 \\
\hline
\end{tabular}

The data in Table I show that the 3D Discrete Shearlet Denoising Algorithm 3DSHEAR is highly competitive against both traditional and other state-of-the-art video denoising algorithm. In particular, 3DSHEAR consistently outperforms the curvelet-based routine 3DCURV, the wavelet-based routines UDWT and DTWT and the 2D shearlet-based algorithm. 3DSHEAR also outperforms or is essentially equivalent to the surfacelets-based denoising algorithm in all cases we tested, except for one case, namely the mobile video sequence for low noise, with standard deviation $\sigma=50$. Notice that for higher noise level 3DSHEAR always provide the best performance in all tests that were run.

Table II: Comparison of running times for different 3D transforms.

\begin{tabular}{|c|c|}
\hline Algorithm & Running time (data size: $192^{3}$ ) \\
\hline \hline SURF & $34 \mathrm{sec}$ \\
\hline 3DSHEAR & $263 \mathrm{sec}$ \\
\hline 2DSHEAR & $154 \mathrm{sec}$ \\
\hline 3D DWT & $7.5 \mathrm{sec}$ \\
\hline
\end{tabular}

The superior performance of the 3DSHEAR algorithm depends in part on its excellent directional selectivity; but it also benefits from the redundancy of the transform, since high redundancy usually produces a better performance in denoising applications. The drawback is that the higher redundancy requires higher computational effort, which explains the worse performance of 3DSHEAR with respect to 3D DWT and SURF in terms of running times. This is reported in the Table II, which compares the running times for these different 3D transforms, applied to a data set of size $193^{3}$; all routines were run using the same system which is based on an Intel CPU $2.93 \mathrm{GHz}$.

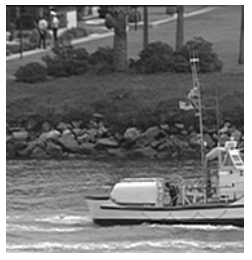

Original frame

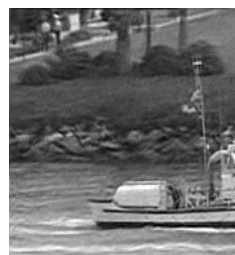

3D SHEAR

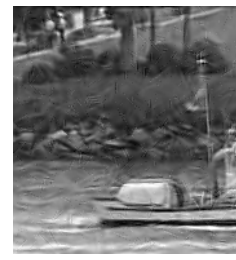

2D SHEAR

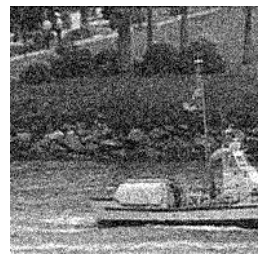

Noisy frame

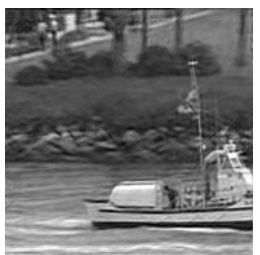

SURF

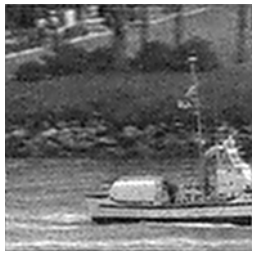

DWT
Fig. 6. Video Denoising of Coast Guard Video Sequence. The figure illustrates the denoising performance on a representative frame of the video sequence using various denoising routines. Starting from the top left: original frame, noisy frame (PSNR=18.62 dB), denoised frame using 3DSHEAR $(\mathrm{PSNR}=27.36 \mathrm{~dB})$, SURF $(\mathrm{PSNR}=26.82 \mathrm{~dB}), 2 \mathrm{DSHEAR}(\mathrm{PSNR}=25.20 \mathrm{~dB})$, DWT $($ PSNR=24.34 dB).

In Fig. 5 and 6, we illustrate the performance of the various video denoising routines on a typical frame extracted from the denoised video sequences Mobile and Coast Guard. Although this type of comparison is more subjective in nature, the figures show that the visual quality of the shearlet-denoised frame is also superior.

\section{B. Video Enhancement}

In several imaging applications, it is important to enhance the visual appearance of certain features that carry useful information. For example, in ultrasound medical images weak edges are usually related to important physical or structural properties so that it is desirable to make weak edges more prominent while keeping the strong features intact. A classical application is mammography, where image enhancement can be useful to improve the visibility of small tumors for early detection [28].

Several techniques have been proposed to enhance the contrast level of an image. For example, since weak edges are mostly associated with the high frequency components of an image, many traditional enhancement methods consist in amplifying the highpass subbands of an image which has been decomposed into different frequency subbands. Unfortunately, these methods are not very efficient to preserve the geometrical features of the data and, as a result, when they are applied 


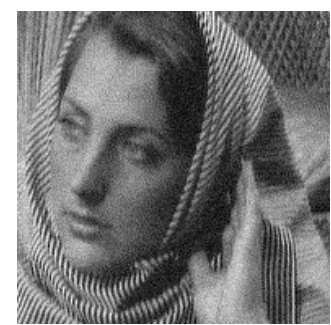

Noisy frame (PSNR 28.16)

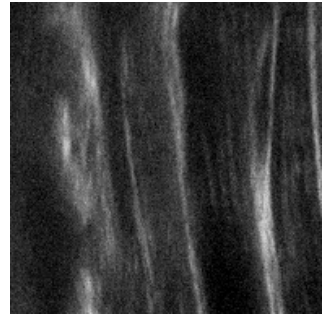

Noisy frame (PSNR 28.16)

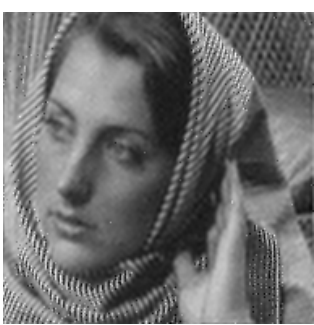

Wavelet-based enhancement

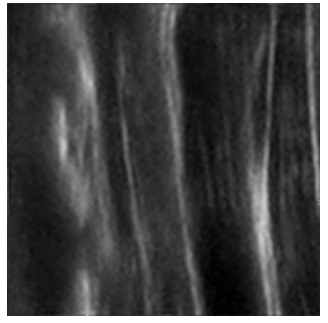

Wavelet-based enhancement

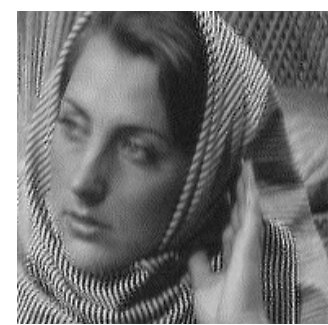

Shearlet-based enhancement

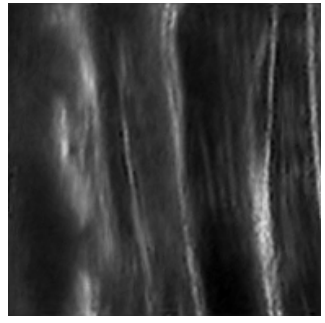

Shearlet-based enhancement

Fig. 7. Video Enhancement. Representative frames from the Barbara video sequence (above) and from the Anterior ultrasound video sequence (below) illustrate the performance of the shearlet-based enhancement algorithm. This is compared against a similar wavelet-based enhancement algorithm.

to enhance weak edges, they also amplify noise and produce visual artifacts.

By contrast, multiscale techniques are much more effective in enhancing weak edges without blowing up the noise [28], [29]. The advantage of the shearlet framework, in particular, is to provide a unique ability to control the geometric information associated with multidimensional data. Thus, shearlets appear to be particularly promising as a tool for selectively enhancing the component of the data associated with the weak edges, as was recently observed in [30] for 2D images (see also [31], [32] for other results concerning the application of directional multiscale transforms in image enhancement). In this section, we present an algorithm which extends this approach to the 3D setting and applies the 3D Discrete Shearlet Transform to decompose data into several directional subbands and to selectively amplify some of the shearlet coefficients. In fact, by the properties of the shearlet decomposition, the shearlet coefficients which are large in magnitude, at fine scales, are closely associated with the singularities of the data. More precisely, strong surfaces of discontinuity will produce large or significant coefficients in all directional subbands, whereas weak surfaces of discontinuity will produce large or significant coefficients only in very few directional subbands. On the other hand, no significantly large coefficients are produced by the noise (provided, of course, SNR is "reasonable").

Based on these observations, each voxel $k$ of a data set $f \in \ell^{2}\left(\mathbb{Z}_{N}^{3}\right)$ can be classified into one of three distinct categories by analysing the magnitude of the corresponding shearlet coefficients $\left\langle f, \psi_{j, \ell, k}^{(d)}\right\rangle$. Notice that heuristic observations have shown that it is sufficient to consider only the shearlet coefficents at the finest scale, so that the parameter $j$ is fixed in the procedure described below. Hence, in our enhancement algorithm, for each voxel $k$, we compute the average and the maximum of the magnitude of the shearlet coefficients taken over all the directional subbands, which we denoted by the functions mean and max, respectively; next, we compute the enhanced coefficient as follows (cf. a similar enhancement is defined in [33]):

$E(x)= \begin{cases}x & \text { if mean }(x) \geq c \sigma, \\ \max \left\{\left(\frac{c \sigma}{|x|}\right)^{p}, 1\right\} x & \text { if mean }(x)<c \sigma \& \max (x) \geq c \sigma, \\ 0 & \text { if } \operatorname{mean}(x)<c \sigma \& \max (x)<c \sigma,\end{cases}$

where $x$ is the input coefficient, $\sigma$ is the standard deviation of the noise in the subband associated with the finest resolution level, $0<p<1$ is a parameter controlling the portion of edges to be treated as considered "weak edges" and $c$ is a tuning parameter, determining the enhancement factor $(c \in[1,5])$.

To illustrate its performance, the shearlet-based enhancement algorithm was tested to enhance two noisy video sequences, the Barbara movie, obtained from the Barbara picture by moving a window frame around the picture) and the Anterior ultrasound movie, showing an ultrasound movie sequence of the Anterior triangle, a muscular region near the neck. In both cases, the noise is additive white Gaussian noise with zero mean and standard deviation $\sigma=10$. Also, in both case the enhancement algorithm was run using parameters $c=1, p=1$, and the performance of the algorithm was compared against an undecimated wavelet-based enhancement routine, which uses the same enhancement function $E$. Representative 2D frames from the enhanced video sequences are illustrated in Figure 7, showing that the shearlet-based routine performs significantly better both in terms of contrast improvement and noise suppression. For comparison with the shearlet-based enhancement, we also run a similar routine based on the surfacelets, but its performance was not better than the wavelet-based routine. This is due in most part to the fact that the surfacelets algorithm has low redundancy, unlike the shearlet-based and wavelet-based algorithms. 
Since the performance of the enhancement algorithm is only partially illustrated by the video frames in Figure 7, to better convince the reader, the complete enhancement videos are available at http://www.math.uh.edu/ dlabate/software.

\section{CONCLUSION}

This paper describes an implementation of the 3D Discrete Shearlet Transform which is based on the directional multiscale framework of the shearlet representation. This implementation faithfully reproduces the frequency decomposition of the 3D shearlet transform which was recently shown to provide optimally sparse approximations for a large class of 3D data. To illustrate the benefits derived from the sparsity of the shearlet representation, our 3D algorithm was tested on problems of video denoising and video enhancement. The performance was found to be extremely competitive against other multiscale methods including wavelets, 3D curvelets and surfaclets. With respect to some of these transforms, the 3D shearlet algorithm has higher redundancy, which accounts for the additional computation effort which requires.

\section{ACKNOWLEDGMENTS}

The authors acknowledge support from NSF grants DMS 1005799 and DMS 1008900.

\section{REFERENCES}

[1] K. Guo, G. Kutyniok, and D. Labate, Sparse Multidimensional Representations using Anisotropic Dilation and Shear Operators, in: Wavelets and Splines, G. Chen, M. Lai (eds.), Nashboro Press, 2006, pp. 189-201.

[2] K. Guo, W.-Q Lim, D. Labate, G. Weiss and E. Wilson, Wavelets with composite dilations, Electron. Res. Announc. Amer. Math. Soc., vol. 10, pp. 78-87, 2004.

[3] E. J. Candès and D. L. Donoho, New tight frames of curvelets and optimal representations of objects with $C^{2}$ singularities, Comm. Pure Appl. Math., vol. 57, no 2, pp. 219-266, 2004.

[4] M. N. Do and M. Vetterli, The contourlet transform: an efficient directional multiresolution image representation, IEEE Trans. Image Process., vol. 14, no. 12, pp. 2091-2106, 2005.

[5] K. Guo, D. Labate, Optimally Sparse Multidimensional Representation using Shearlets, SIAM J. Math. Anal., vol 39, no. 1, pp. 298-318, 2007.

[6] D. L. Donoho, M. Vetterli, R. A. DeVore, and I. Daubechies, Data compression and harmonic analysis, IEEE Trans. Inform. Th., vol. 44, pp. 2435-2476, 1998.

[7] G. R. Easley, D. Labate, F. Colonna, Shearlet-Based Total Variation Diffusion for Denoising, IEEE Trans. Image Process., vol. 18, no. 2, pp. 260-268, 2009.

[8] G. R. Easley, D. Labate, and W. Lim, Sparse Directional Image Representations using the Discrete Shearlet Transform, Appl. Comput. Harmon. Anal., vol. 25, no. 1, pp. 25-46, 2008.

[9] S. Yi, D. Labate, G. R. Easley, and H. Krim, A Shearlet approach to Edge Analysis and Detection, IEEE Trans. Image Process., vol. 18, no. 5, pp. 929-941, 2009.

[10] F. Colonna, G. Easley, K. Guo, and D. Labate, Radon Transform Inversion using the Shearlet Representation, Appl. Comput. Harmon. Anal., vol. 29, no. 2, pp. 232-250, 2010.

[11] P. Kittipoom, G. Kutyniok, and W.-Q Lim, Construction of Compactly Supported Shearlet Frames, Constr. Approx., vol. 35, pp. 21-72, 2012.

[12] G. Kutyniok and W.-Q Lim, Compactly Supported Shearlets are Optimally Sparse, J. Approx. Theory, vol. 63, pp. 1564-1589, 2011.

[13] E. J. Candès, L. Demanet, D. L. Donoho and L. Ying, Fast discrete curvelet transforms, SIAM Multiscale Model. Simul., vol. 5, no. 3, pp. 861-899, 2006.

[14] Y. Lu and M. N. Do, Multidimensional Directional Filter Banks and Surfacelets, IEEE Trans. Image Process., vol. 16, no. 4, pp. 918-931, 2007.
[15] K. Guo, and D. Labate, Optimally Sparse 3D Approximations using Shearlet Representations,Electron. Res. Announc. Amer. Math. Soc., vol. 17, pp. 126-138, 2010.

[16] K. Guo, and D. Labate, Optimally Sparse Representations of 3D Data with $C^{2}$ Surface Singularities using Parseval Frames of Shearlets, preprint 2010 .

[17] E. L. Pennec and S. Mallat, Sparse geometric image representations with bandelets, IEEE Trans. Image Process., vol. 14, no. 4, pp. 423438, 2005.

[18] S. Mallat, Geometrical grouplets, Appl. Comput. Harmon. Anal., vol. 26, no. 2, pp. 161-180, 2009.

[19] K. Guo, and D. Labate, The Construction of Smooth Parseval Frames of Shearlets, preprint 2011.

[20] P. J. Burt, E. H. Adelson, The Laplacian pyramid as a compact image code, IEEE Trans. Commun., vol. 31, no. 4, pp. 532-540, 1983.

[21] A. Averbuch, R. R. Coifman, D. L. Donoho, M. Israeli, and Y. Shkolnisky, A framework for discrete integral transformations I - The pseudopolar Fourier transform, SIAM Journal on Scientific Computing, vol. 30 , no. 2, pp. 764-784, 2008.

[22] Y. Keller, A. Averbuch, Y. Shkolnisky, Algebraically accurate volume registration using Euler's theorem and the 3D pseudopolar FFT , IEEE Comput. Soc. Conf. on Comput. Vision and Pattern Rec., vol. 2, pp. 795-800, 2005

[23] D. L. Donoho and I. M. Johnstone, Ideal spatial adaptation by wavelet shrinkage, Biometrika vol. 81, no. 3, pp. 425455, 1994.

[24] D. L. Donoho, Denoising via soft thresholding, IEEE Trans. Inform. Th., vol. 41, no. 3, pp. 613-627, 1995

[25] G. Chang, B. Yu and M. Vetterli, Adaptive Wavelet Thresholding for Image Denoising and Compression, IEEE Trans. Image Process., vol. 9, no. 9 , pp. 1532-1546, 2000.

[26] J. L. Starck, F. Murtagh, J. Fadili, Sparse image and signal processing: wavelets, curvelets, morphological diversity, Cambridge U. Press, 2010.

[27] I. W. Selesnick, The double-density dual-tree DWT, IEEE Trans. Signal Process., vol. 52, no. 5, pp. 13041314, 2004.

[28] C. Chang, A. F. Laine, Coherence of Multiscale Features for Contrast Enhancement of Digital Mammograms, IEEE Trans. Info. Tech. in Biomedicine, vol. 3, no. 1, pp. 32-46, 1999.

[29] S. Dippel, M. Stahl, R. Wiemker, and T. Blaffert, Multiscale contrast enhancement for radiographies: Laplacian pyramid versus fast wavelet transform, IEEE Trans. Medical Imaging, vol. 21, no. 4, pp. 343-353, 2002.

[30] V. M. Patel, G. R. Easley, and D. M. Healy, A new multiresolution generalized directional filter bank design and application in image enhancement, Proc. IEEE Int. Conf. on Image Process., San Diego, pp. 2816-2819, 2008.

[31] J. L. Starck, F. Murtagh, E. J. Candès, and D. L. Donoho, Gray and color image contrast enhancement by the curvelet transform, IEEE Trans. Image Process., vol. 12, no. 6, pp. 706-717, 2003.

[32] J. Zhou, A. L. Cunha, and M. N. Do, Non-subsampled Contourlet transform: construction and application in Enhancement, IEEE Int. Conf. on Image Process., Genoa, 2005

[33] K. V. Velde, Multi-scale color image enhancement, Proc.IEEE Int. Conf. on Image Process., vol. 3, pp. 584-587, 1999.

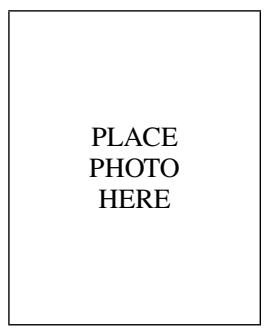

Pooran S. Negi Pooran Singh Negi received the M.S. in Mathematics from the University of Houston in 2008. He is currently pursuing the Ph.D. degree in the Mathematics from the University of Houston. His research interests include high-dimensional signal processing and applied harmonic analysis.

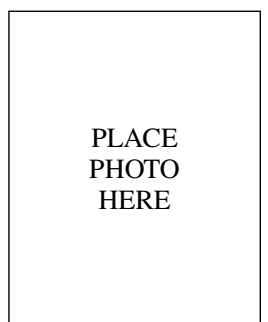

Demetrio Labate received the Ph.D. degree in electrical engineering from the Politecnico di Torino, Italy, in 1995, and the Ph.D. degree in mathematics from the Georgia Institute of Technology, Atlanta, in 2000. He was a Chauvenet lecturer at Washington University in St.Louis and an Assistant Professor at NC State University. Since 2009, he is Associate Professor of Mathematics at the University of Houston. His research interests include harmonic analysis, wavelet theory, time-frequency analysis and sparse representations. He received the NSF CA-

REER Award in Applied Mathematics in 2008. 